\title{
GRB 190114C: from prompt to afterglow?
}

\author{
M. E. Ravasio ${ }^{1,2}$, G. Oganesyan ${ }^{3,4}$, O. S. Salafia ${ }^{1,2}$, G. Ghirlanda ${ }^{1,2}$, G. Ghisellini ${ }^{1}$, M. Branchesi ${ }^{3,4}$, \\ S. Campana ${ }^{1}$, S. Covino $^{1}$, and R. Salvaterra ${ }^{5}$
}

\author{
1 INAF - Osservatorio Astronomico di Brera, Via E. Bianchi 46, 23807 Merate, Italy \\ e-mail: m.ravasio5@campus.unimib.it \\ 2 Dipartimento di Fisica G. Occhialini, Univ. di Milano Bicocca, Piazza della Scienza 3, 20126 Milano, Italy \\ 3 Gran Sasso Science Institute, Viale F. Crispi 7, 67100 L'Aquila, Italy \\ ${ }^{4}$ INFN - Laboratori Nazionali del Gran Sasso, 67100 L'Aquila, Italy \\ 5 INAF - Istituto di Astrofisica Spaziale e Fisica Cosmica, Via E. Bassini 15, 20133 Milano, Italy
}

Received 5 February 2019 / Accepted 23 April 2019

\begin{abstract}
GRB 190114C is the first gamma-ray burst detected at very high energies (VHE, i.e., $>300 \mathrm{GeV}$ ) by the MAGIC Cherenkov telescope The analysis of the emission detected by the Fermi satellite at lower energies, in the $10 \mathrm{keV}-100 \mathrm{GeV}$ energy range, up to $\sim 50 \mathrm{~s}$ (i.e., before the MAGIC detection) can hold valuable information. We analyze the spectral evolution of the emission of GRB 190114C as detected by the Fermi Gamma-Ray Burst Monitor (GBM) in the $10 \mathrm{keV}-40 \mathrm{MeV}$ energy range up to $\sim 60 \mathrm{~s}$. The first $4 \mathrm{~s}$ of the burst feature a typical prompt emission spectrum, which can be fit by a smoothly broken power-law function with typical parameters. Starting on $\sim 4$ s post-trigger, we find an additional nonthermal component that can be fit by a power law. This component rises and decays quickly. The $10 \mathrm{keV}-40 \mathrm{MeV}$ flux of the power-law component peaks at $\sim 6 \mathrm{~s}$; it reaches a value of $1.7 \times 10^{-5} \mathrm{erg} \mathrm{cm}^{-2} \mathrm{~s}^{-1}$. The time of the peak coincides with the emission peak detected by the Large Area Telescope (LAT) on board Fermi. The power-law spectral slope that we find in the GBM data is remarkably similar to that of the LAT spectrum, and the GBM+LAT spectral energy distribution seems to be consistent with a single component. This suggests that the LAT emission and the power-law component that we find in the GBM data belong to the same emission component, which we interpret as due to the afterglow of the burst. The onset time allows us to estimate that the initial jet bulk Lorentz factor $\Gamma_{0}$ is about 500, depending on the assumed circum-burst density.
\end{abstract}

Key words. radiation mechanisms: non-thermal - gamma-ray burst: individual: GRB 190114C - gamma-ray burst: general

\section{Introduction}

Soon after its launch, the Fermi satellite has been detecting ${ }^{1}$ about 14 gamma-ray bursts (GRBs) per year on average with its Large Area Telescope (LAT) in the high-energy (HE) range between a few MeV to $100 \mathrm{GeV}$ (Ackermann et al. 2013). The Fermi/LAT GRBs confirm the detections by the Astro Rivelatore Gamma ad Immagini Leggero (Agile/GRID - Giuliani et al. 2008, 2010; Del Monte et al. 2011) and the earlier results of the Compton Gamma Ray Observatory/EGRET (Sommer et al. 1994; Hurley et al. 1994; González et al. 2003). Until very recently, observations of GRBs emission at very high energies (VHE) by Imaging Atmospheric Cherenkov Telescopes (IACT) resulted only in upper limits (Aliu et al. 2014; Carosi et al. 2015; Hoischen et al. 2017). GRB 190114C is the first burst detected at $>300 \mathrm{GeV}$ by the Major Atmospheric Gamma Imaging Cherenkov Telescopes (MAGIC; Mirzoyan et al. 2019).

Gammy-ray burst emission in the $100 \mathrm{MeV}-100 \mathrm{GeV}$ energy range as detected by LAT typically starts with a short delay with respect to the trigger time of the $\mathrm{keV}-\mathrm{MeV}$ component (Omodei 2009; Ghisellini et al. 2010; Ghirlanda et al. 2010) and extends until after the prompt emission. This behavior has also been observed in short GRBs (Ghirlanda et al. 2010; Ackermann et al. 2010). While the early HE emission (simultaneous with the $\mathrm{keV}-\mathrm{MeV}$ component) shows some variability, its long-lasting tail decays smoothly. A possible transition from

\footnotetext{
1 http://fermi.gsfc.nasa.gov/ssc/observations/types/ grbs/lat_grbs/table.php
}

an early steep decay $\left(\propto t^{-1.5}\right)$ to a shallower regime $\left(\propto t^{-1}\right)$ has been reported (Ghisellini et al. 2010; Ackermann et al. 2013) and a faster temporal decay in brighter bursts has been claimed (Panaitescu 2017).

During the prompt emission phase (as detected, e.g., by the Gamma Ray Burst Monitor, GBM, on board the Fermi satellite), the LAT spectrum can either be the extension above $100 \mathrm{MeV}$ of the typical sub-MeV GRB spectrum (which is usually fitted with the Band function; Band et al. 1993), or it requires an additional spectral component in the form of a power law (PL), as in GRB 080916C, 110713A (Ackermann et al. 2013), 090926A (Yassine et al. 2017), and 130427A (Ackermann et al. 2014). In a few bursts, this additional PL component has been found to extend to the X-ray range ( $<20 \mathrm{keV}$; e.g., 090510 , Ackermann et al. 2010, and 090902B, Abdo et al. 2009). When the prompt emission has ceased, the LAT spectrum is often fit by a PL with photon index $\Gamma_{\mathrm{PL}} \sim-2$.

The interpretation of the HE emission of GRBs is still debated (see Nava 2018 for a review). It has been proposed that the LAT emission that extends after the end of the prompt emission is the afterglow that is produced in the external shock that is driven by the jet into the circum-burst medium (Kumar \& Barniol Duran 2009, 2010; Ghisellini et al. 2010). The mechanism that causes this might be synchrotron emission. The correlation of the LAT luminosity with the prompt emission energy (Nava et al. 2014) and the direct modeling of the broadband spectral energy distribution (initially in a few bursts, Kumar \& Barniol Duran 2009, 2010 and then in a larger sample 
Beniamini et al. 2015) support the hypothesis of a synchrotron origin.

A possible problem with the synchrotron interpretation are VHE photons (tens of $\mathrm{GeV}$ ), which exceed the theoretical limit of synchrotron emission from shock-accelerated electrons. This limit is $\sim 70 \mathrm{MeV}$ in the comoving frame (Guilbert et al. 1983, see also de Jager et al. 1996; Lyutikov 2010 for a lower value of about $30 \mathrm{MeV}$ ), but downstream magnetic field stratification (Kumar et al. 2012) or acceleration in magnetic reconnection layers (Uzdensky et al. 2011; Cerutti et al. 2013) can alleviate this apparent discrepancy.

The deceleration of the jet by the interstellar medium is expected to produce a peak in the afterglow light curve at a time $t_{\mathrm{p}}$ that corresponds to the transition from the coasting to the deceleration phase (Sari \& Piran 1999). $t_{\mathrm{p}}$ depends on the blast wave kinetic energy $E_{\mathrm{k}}$, on the density of the circumburst medium (and its radial profile), and on the initial bulk Lorentz factor $\Gamma_{0}$ (representing the maximum velocity that the jet attained, i.e., that of the coasting phase). Therefore, by deducing $E_{\mathrm{K}}$ from the prompt emission and making an assumption on the circum-burst medium density, it is possible to estimate $\Gamma_{0}$ (Molinari et al. 2007; Ghirlanda et al. 2012, 2018) for large samples of GRBs.

If the $\mathrm{GeV}$ component is afterglow produced by the external shock, the time $t_{\mathrm{p}}$ provides an estimate of $\Gamma_{0}$ (see also Nava et al. 2017), as shown for the first time in the case of the LATdetected GRB 090510 (Ghirlanda et al. 2010). The shorter $t_{\mathrm{p}}$, the larger $\Gamma_{0}$ : LAT bursts have the shortest times $t_{\mathrm{p}}$ (Ghirlanda et al. 2018) and therefore provide the highest values of $\Gamma_{0}$ up to 1200 (GRB 090510 - Ghirlanda et al. 2018). As discussed in Ghisellini et al. (2010), this might indicate that a large $\Gamma_{0}$ helps to accelerate very high energy electrons, which emit at high photon energies. Furthermore, even a small fraction of photons of the prompt phase can be scattered by the circum-burst medium and act as targets for the $\gamma-\gamma$ to $e^{ \pm}$process: this enhances the lepton abundance of the medium, thus making shock acceleration of the leptons more efficient (Beloborodov 2005; Ghisellini et al. 2010).

While the LAT emission, which in some cases is detected up to hours after the end of the prompt, seems to be of external origin, a possible challenge is the interpretation of the early LAT emission that is detected during the prompt phase. It has been argued (Zhang et al. 2011; He et al. 2011) that the very early LAT emission has an internal origin (Bošnjak et al. 2009) because it can be due to inverse Compton-scattered synchrotron photons of the prompt (SSC). The delay of the $\mathrm{GeV}$ emission as measured by LAT could be explained by inverse Compton emission that occurred in the Klein-Nishina regime at early times (Daigne 2012; Bošnjak et al. 2009). While recent findings seem to support a synchrotron origin of $\mathrm{keV}-\mathrm{MeV}$ photons (Oganesyan et al. 2017, 2018; Ravasio et al. 2018), the presence of a soft excess $(<50 \mathrm{keV})$ that is clearly detected so far in GRB 090902B (Abdo et al. 2009), GRB 090510 (Ackermann et al. 2010), and GRB 090926A (Yassine et al. 2017), represents a challenge for the SSC interpretation (but see Toma et al. 2011) and would be more easily interpreted as the low-energy extension of the $\mathrm{GeV}$ afterglow component.

This paper is based on the study of the emission of GRB 190114C (Sect. 2) as detected by the GBM in the $10 \mathrm{keV}-$ $40 \mathrm{MeV}$ energy range, up to $61 \mathrm{~s}$ after the trigger. We also consider data from the Burst Alert Telescope (BAT) and the X-Ray Telescope (XRT) on board the Neil Gehrels Swift Observatory in three time intervals. While the properties of GRB 190114C are similar to other bursts detected by LAT, emission that might extend up to the TeV energy range as detected by MAGIC (Mirzoyan et al. 2019) makes this event unique so far. Data extraction and analysis are presented in Sect. 3 and in Sect. 4, where we show the appearance and temporal evolution of a nonthermal power-law spectral component starting from $4 \mathrm{~s}$ after the trigger. In Sect. 5 we discuss our results and their implications.

\section{GRB $190114 C$}

On 14 January 2019 at 20:57:03 UT, both the Fermi/GBM and the Swift/BAT were triggered by GRB 190114C (Hamburg et al. 2019; Gropp et al. 2019). The burst was also detected in hard X-rays by the SPI-ACS instrument on board INTEGRAL, with evidence for long-lasting emission (Minaev \& Pozanenko 2019), by the Mini-CALorimeter (MCAL) instrument on board the AGILE satellite (Ursi et al. 2019), by the Hard X-ray Modulation Telescope (HXMT) instrument on board the Insight satellite (Xiao et al. 2019), and by Konus-Wind (Frederiks et al. 2019).

Remarkably, this burst was the first to be detected at very high energies by a Cherenkov telescope: MAGIC was able to point the source $50 \mathrm{~s}$ after the Swift trigger, revealing the burst with a significance $>20 \sigma$ at energies $>300 \mathrm{GeV}$ (Mirzoyan et al. 2019). The burst was also detected by LAT. It remained in its field of view until $150 \mathrm{~s}$ after the GBM trigger (Kocevski et al. 2019).

The redshift was first measured by the Nordic Optical Telescope (NOT; Selsing et al. 2019) (soon confirmed by the Gran Telescopio Canarias, GTC; Castro-Tirado et al. 2019), with the value $z=0.4245 \pm 0.0005$.

The fluence (integrated in the $10-1000 \mathrm{keV}$ energy range) measured by the GBM is $3.99 \times 10^{-4} \pm 8 \times 10^{-7} \mathrm{erg} \mathrm{cm}^{-2}$ and the peak photon flux (with $1 \mathrm{~s}$ binning in the same energy range) is $246.86 \pm 0.86 \mathrm{~cm}^{-2} \mathrm{~s}^{-1}$ (Hamburg et al. 2019). As reported in Hamburg et al. (2019), the corresponding isotropic equivalent energy and luminosity are $E_{\text {iso }} \sim 3 \times 10^{53}$ erg and $L_{\text {iso }} \sim 1 \times$ $10^{53} \mathrm{erg} \mathrm{s}^{-1}$, respectively. These values make this burst consistent with the $E_{\text {peak }}-E_{\text {iso }}$ (Amati et al. 2002) and $E_{\text {peak }}-L_{\text {iso }}$ (Yonetoku et al. 2004) correlations (Frederiks et al. 2019).

The prompt emission of GRB 190114C is characterized by a first (multi-peaked) pulse that lasted $\sim 5.5 \mathrm{~s}$, followed by a second weaker and softer pulse from 15 to $22 \mathrm{~s}$ after trigger (as shown in the top panel of Fig. 1), and then a weaker and long tail that lasted up to some hundreds of seconds (Hamburg et al. 2019; Minaev \& Pozanenko 2019).

\section{Data analysis}

\subsection{Fermi/GBM}

The GBM is composed of 12 sodium iodide (NaI, $8 \mathrm{keV}-1 \mathrm{MeV}$ ) and 2 bismuth germanate (BGO, $200 \mathrm{keV}-40 \mathrm{MeV}$ ) scintillation detectors (Meegan et al. 2009). We analyzed the data of the three brightest $\mathrm{NaI}$ detectors with a viewing angle smaller than $60^{\circ}$ (n3, n4, and n7) and both the BGO detectors (b0 and b1). In particular, we selected the energy channels in the range $8-900 \mathrm{keV}$ for $\mathrm{NaI}$ detectors, excluding the channels in the range $25-40 \mathrm{keV}$ because of the iodine $\mathrm{K}$-edge at $33.17 \mathrm{keV}^{2}$ and $0.3-40 \mathrm{MeV}$ for BGO detectors. Spectral data files and the corresponding response matrix files (.rsp2) were obtained from the online archive $^{3}$, and the spectral analysis was performed with the public

\footnotetext{
2 https://fermi.gsfc.nasa.gov/ssc/data/analysis/GBM_ caveats.html

3 https://heasarc.gsfc.nasa.gov/W3Browse/fermi/

fermigbrst.html
} 
software RMFIT- (v. 4.3.2). To model the background, we selected background spectra in time intervals well before and after the burst ( $\approx-130:-10 \mathrm{~s}$ and $210: 370 \mathrm{~s}$ from the trigger time) and modeled them with a polynomial function up to the third order. We used time-tagged event (TTE) data, and rebinned them with a time resolution of $0.3 \mathrm{~s}$ during the first emission episode of the burst. After the first emission episode, we rebinned the data in progressively longer time bins up to the second minor peak of the light curve (from $\sim 15 \mathrm{~s}$ to $\sim 23 \mathrm{~s}$ ), which was analyzed as a single bin. Finally, we analyzed the $23-61$ s time interval as two consecutive time bins (23-47 s and 47-61 s).

\subsection{Swift: BAT and XRT data}

We also considered BAT data extracted for three time bins, 6-6.3 s, 47-61 s, and 87-232 s, both as a check of the consistency with the parameters of the fit obtained in the same time intervals from GBM data and as a way to extend our analysis to later times. We downloaded BAT event files from the Swift data archive $^{4}$. To extract BAT spectra, we used the latest version of the HEASOFT package (v6.25). We generated BAT spectral files with the batbinevt task, applying the correction for systematic errors with the batupdatephakw and batphasyserr tasks. We generated response files with the batdrmgen tool. We adopted the latest calibration files (CALDB release 2017-10-16).

In addition, we retrieved XRT event files from the Swift/XRT archive ${ }^{5}$. The source and background files were extracted with the xselect tool. We removed the central region of the XRT image to avoid pile-up effects, following the procedure described in Romano et al. (2006). We generated an ancillary response file with the xrtmkarf task. We excluded all the channels below $1.5 \mathrm{keV}$ because an apparent low-energy excess has been reported in Beardmore (2019). We then rebinned the energy channels using the grppha tool, requiring at least 40 counts per bin.

We used the multiplicative XSPEC models tbabs and ztbabs to account for Galactic and intrinsic absorption of the X-ray spectrum by neutral hydrogen (Wilms et al. 2000). The value of Galactic neutral hydrogen column density in the direction of GRB 190114C was found from Kalberla et al. (2005). The intrinsic column density $7.7 \times 10^{22} \mathrm{~cm}^{-2}$ was estimated by fitting the late-time X-ray spectrum $\left(5.6 \times 10^{4}-5.7 \times 10^{5} \mathrm{~s}\right)$.

\subsection{Fitting models}

A preliminary analysis of the GBM spectrum was reported in Hamburg et al. (2019): the time-integrated spectrum from 0 to $38.59 \mathrm{~s}$ (which includes the two pulses of the burst but also the inter-pulse interval) was fit with a Band function, finding $E_{\text {peak }}=$ $998.6 \pm 11.9 \mathrm{keV}, \alpha=-1.058 \pm 0.003$, and $\beta=-3.18 \pm 0.07$. In addition, the authors also reported a strong statistical preference for an extra power-law component.

In our time-resolved analysis, we fit the spectra with a smoothly broken power-law (SBPL, see Ravasio et al. 2018 for a description of the functional form). The SBPL is one of the empirical functions that is generally used to model GRB spectra (Kaneko et al. 2006; Gruber et al. 2014). The SBPL is made of two power laws, with spectral indices $\alpha$ and $\beta$, which are smoothly connected at the break energy (usually corresponding

\footnotetext{
4 http://heasarc.gsfc.nasa.gov/cgi-bin/W3Browse/ swift.pl

5 http://www.swift.ac.uk/archive/
}

to the $v F_{v}$ peak of the spectrum, $\left.E_{\text {peak }}\right)$. As in Ravasio et al. (2018), the curvature parameter was kept fixed at $n=2$.

Because an additional power-law component was reported in Hamburg et al. (2019), we also added an additional power-law component in the fitting procedure, with two free parameters, the normalization $N$ and the spectral index $\Gamma_{\mathrm{PL}}$.

\section{Results}

Figure 1 shows the results of the time-resolved spectral analysis of GBM data. We find that all spectra belonging to the first emission episode (from $0 \mathrm{~s}$ to $4.8 \mathrm{~s}$ ) are reasonably well fit by an SBPL model and no additional power-law component is required. The low- and high-energy spectral indices of the SBPL model are shown in panel C of Fig. 1 (red and black symbols, respectively). Their values are consistent with the typical distributions obtained from the analysis of large samples of GBM bursts (Goldstein et al. 2012; Gruber et al. 2014; Nava et al. 2011; Kaneko et al. 2006). The peak energy (panel D in Fig. 1) evolves and tracks the flux of the light curve, with an average value of $E_{\text {peak }}=510 \pm 170 \mathrm{keV}$.

The additional power-law component starts in the 4.8-5.4 s and 5.4-6.0 s time bins, where the superposition of an SBPL and a PL component is preferred over the SBPL component alone (an F-test yields a 6 and $7.5 \sigma$ preference for the SBPL+PL model in the first and second bin, respectively).

The power-law component reaches its peak in the time bin $6-6.3 \mathrm{~s}$, with a flux of $1.7 \pm 0.2 \times 10^{-5} \mathrm{erg} \mathrm{cm}^{-2} \mathrm{~s}^{-1}$, integrated in the energy range $10 \mathrm{keV}-40 \mathrm{MeV}$. From $6.3 \mathrm{~s}$ onward, the spectrum is well fit ( $p$-value $>0.3$ in all bins) by a single power-law PL component, with no increase in the goodness of fit when the SBPL component is added. Moreover, when we tried to fit with the SBPL function, the peak energy $E_{\text {peak }}$ was completely unconstrained, and the values found for the two spectral indices $\alpha$ and $\beta$ are consistent with each other within the errors. The single power-law spectral slope is shown by the blue symbols in panel $\mathrm{C}$ of Fig. 1. Its $10 \mathrm{keV}-40 \mathrm{MeV}$ flux is shown by the blue symbols in panel B.

The average spectral slope of the PL component in the time interval $4.8-15.3 \mathrm{~s}$ is $\Gamma_{\mathrm{PL}}=-1.81 \pm 0.08$, similar to the spectral slope found in the LAT data (at $>100 \mathrm{MeV}$, Kocevski et al. 2019) in the same time interval (Wang et al. 2019). After $\sim 10 \mathrm{~s}$, the slope of the power law becomes constant and settles at the -2 value, again similar to the LAT index. The second emission episode was fit by an SBPL, with $\alpha=-1.51 \pm 0.06$, $\beta=-2.33 \pm 0.06$, and $E_{\text {peak }}=63 \pm 3 \mathrm{keV}$. The parameters of the additional power law were not constrained, and the fit did not improve when it was included. After $22.8 \mathrm{~s}$, the spectrum was again well fit by a power law alone, with index $\Gamma_{\mathrm{PL}} \sim-2$. The flux of the PL component (panel B of Fig. 1) decayed steeply from the peak up to $15 \mathrm{~s}$ (a reference green line $\propto t^{-2.8}$ is shown). From $15-50 \mathrm{~s}$, the temporal decay of the flux was consistent with $t^{-1.0}$.

We also added BAT data for the time intervals 6.0-6.3 $\mathrm{s}$ and 11-14 s. In both time bins, BAT+GBM data were fit together with a single PL, from which we obtained best-fit parameters that were consistent with the analysis of GBM data alone. We also verified that BAT data alone for the first time bin result in power-law parameters that were fully consistent with those derived from the fit of the GBM spectrum alone. Figure 2 shows the spectral energy distribution of the three time intervals (as labeled). Spectral data used in the fits are BAT+GBM for interval 6-6.3 s and 11-14 s. XRT+BAT+GBM spectra are shown for the last time bin (66-92 s). Wang et al. (2019) analyzed the LAT 




Fig. 1. Spectral evolution of GRB 190114C. Two spectral components are shown: smoothly broken power law (SBPL, red symbols) and power law (PL, blue circles). $1 \sigma$ errors are shown. Panel A: count rate light curve (black solid line for GBM NaI detector 3 and purple solid line for GBM BGO detector 0). Panel B: flux (integrated in the $10 \mathrm{keV}-40 \mathrm{MeV}$ energy range) of the two spectral components. The green line is a power law with slope -2.8 up to $15 \mathrm{~s}$, with slope -1 when the decay of the flux is shallower. Panel $C$ : temporal evolution of the spectral photon index of the SBPL (red and black symbols) and of the PL (blue symbols). Panel D: evolution of the peak energy $\left(E_{\text {peak }}\right)$ of the SBPL model.

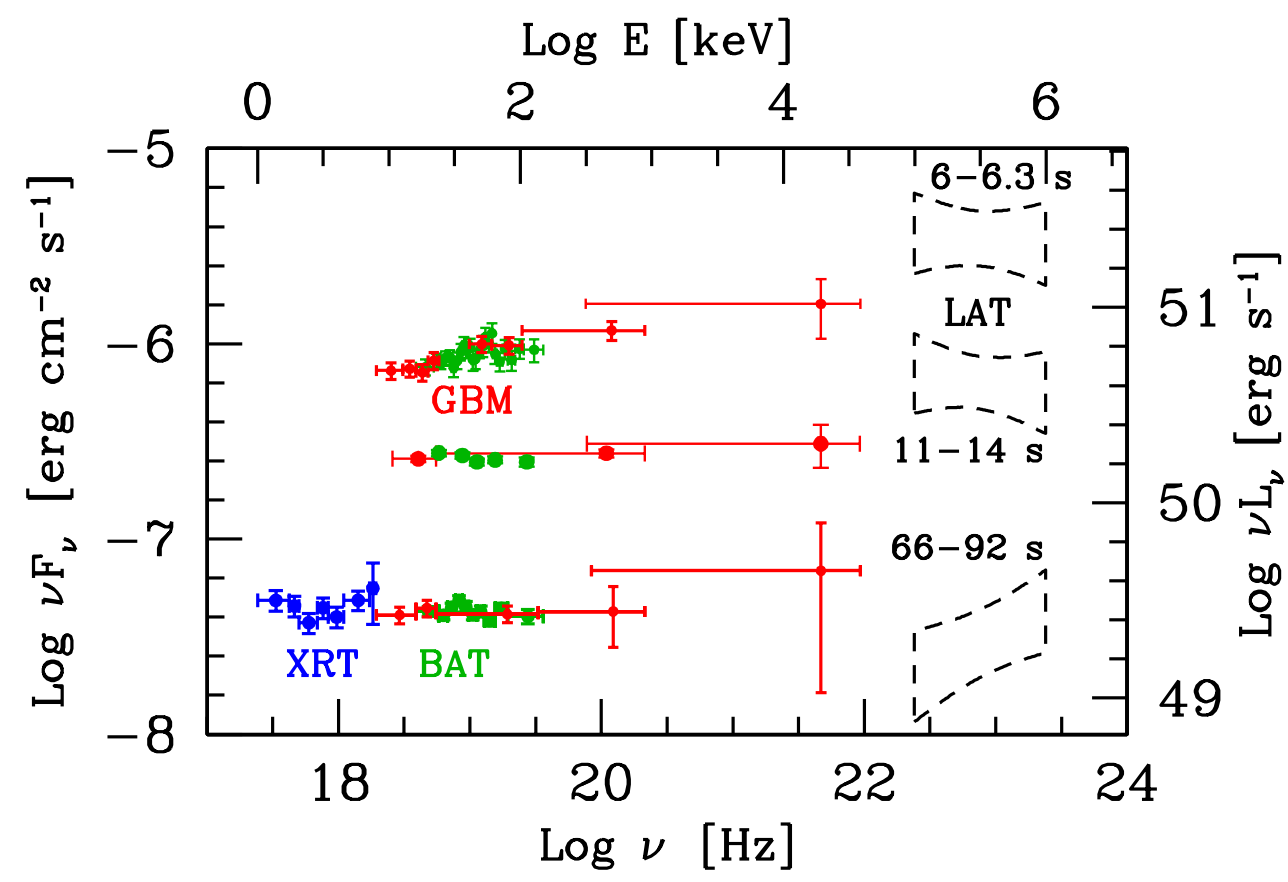

Fig. 2. $\mathrm{X}$-ray to $\mathrm{GeV}$ SED of GRB 190114C at three specific times: at 6-6.3 s, when the power-law component peaks in the GBM data (see panel B of Fig. 1, blue symbols), at 11-14 s, and at 66-92 s (as labeled). We show the GBM, BAT, and XRT data (the latter deabsorbed, as described in the text). Errors and upper limits on the data points represent $1 \sigma$. The LAT butterflies represent the range of fluxes and indices of the power law reported in the analysis of Wang et al. (2019). spectrum of GRB 190114C by fitting the high-energy data with a power-law model. Figure 2 also shows the LAT flux and spectral index with butterflies (including the corresponding uncertainties) for the same time intervals, to be compared with our results.

The GBM and BAT data appear to be connect to the LAT emission, as analyzed by Wang et al. (2019). In the two time intervals $6-6.3 \mathrm{~s}$ and $11-14 \mathrm{~s}$, the photon indices of the LAT spectrum are $\Gamma_{\mathrm{PL}}=-2.06 \pm 0.30$ and $\Gamma_{\mathrm{PL}}=-2.10 \pm 0.31$, respectively, which are consistent with the values we obtained from our analysis. The LAT emission is slightly higher than the GBM extrapolation (by less than 60\%: less than $2 \sigma$ ). Moreover, we analyzed XRT+BAT+GBM data from $66 \mathrm{~s}$ to $92 \mathrm{~s}$ to check again for consistency with the LAT flux given in Wang et al. (2019) and also to track the power-law evolution at later times. As shown in Fig. 2, the LAT flux is still consistent with extrapolation of the joint XRT+BAT+GBM data fit. From our analysis, the fit of XRT+BAT+GBM data from $66 \mathrm{~s}$ to $92 \mathrm{~s}$ with a PL function results in a spectral slope $\Gamma_{\mathrm{PL}}=-2.01 \pm 0.05$, 
which is only marginally consistent with the values obtained by Wang et al. (2019) for the LAT data $\left(\Gamma_{\mathrm{PL}}=-1.67 \pm 0.27\right)$. We note, however, that the spectral slopes reported in Wang et al. (2019) have large uncertainties and show a rapid variability. In summary, Fig. 2 shows that the keV-MeV and $\mathrm{GeV}$ emissions have a similar time decay and similar slopes, suggesting that they belong to the same component. However, because of the uncertainties on the LAT spectral parameters reported in Wang et al. (2019), the possibility that the GeV and keV-MeV data belong to two different components cannot be excluded.

Several slightly different formulae can be used to derive the bulk Lorentz factor $\Gamma_{0}$ of the coasting phase from the observational data. The required parameters are (i) the peak time of the light curve $t_{\mathrm{p}}$; (ii) the isotropic equivalent kinetic energy of the jet $E_{\mathrm{K}}$ after the emission of the prompt radiation; (iii) the circumburst density $n$, which is responsible for the deceleration of the jet, and (iv) its radial profile.

Usually, it is assumed that the observed isotropic equivalent energy radiated in the prompt phase $E_{\text {iso }}$ is a fraction $\eta$ of the kinetic energy, implying $E_{\mathrm{K}}=E_{\mathrm{iso}} / \eta$, typically with $\eta=0.1$ or 0.2 . The density is assumed to have a radial profile $n \propto R^{-s}(R$ is the distance from the central engine originating the GRB). We considered the case of a uniform density $(s=0)$, or a steady stellar wind density profile $(s=2)$. In the latter case, the density depends on the mass rate $\dot{M}_{\mathrm{w}}$ of the wind and its velocity $v_{\mathrm{w}}$ (Chevalier \& Li 2000), $n(R)=\dot{M}_{\mathrm{w}} /\left(4 \pi v_{\mathrm{w}} R^{2} m_{\mathrm{p}}\right)$.

The different formulae used to calculate $\Gamma_{0}$ have been thoroughly discussed in Ghirlanda et al. (2018). As in that paper, we used the formula derived in Nava et al. (2013)

$\Gamma_{0}=\left[\frac{(17-4 s)(9-2 s) 3^{2-s}}{2^{10-2 s} \pi(4-s)}\left(\frac{E_{\mathrm{K}}}{n_{0} m_{\mathrm{p}} c^{5-s}}\right)\right]^{\frac{1}{8-2 s}} t_{\mathrm{p}, z^{-2 s}}^{\frac{3-s}{8-2 s}}$,

which for the two different cases of homogeneous medium $(s=$ $0)$ and wind density profile $(s=2)$, becomes

$\Gamma_{0} \propto\left(\frac{E_{\mathrm{iso}}}{\eta n_{0} m_{\mathrm{p}} c^{5}}\right)^{\frac{1}{8}} t_{\mathrm{p}, z}^{-\frac{3}{8}} \quad(s=0)$

$\Gamma_{0} \propto\left(\frac{E_{\text {iso }}}{\eta n_{0} m_{\mathrm{p}} c^{3}}\right)^{\frac{1}{4}} t_{\mathrm{p}, z}^{-\frac{1}{4}} \quad(s=2)$.

Here $t_{\mathrm{p}}$ is measured in the source cosmological rest frame, that is, $t_{\mathrm{p}, z}=t_{\mathrm{p}} /(1+z), m_{\mathrm{p}}$ is the mass of the proton, and $n_{0}$ is the normalization of the circum-burst density profile, that is, $n(R)=n_{0} R^{-s}$.

Assuming $E_{\text {iso }}=2.6 \times 10^{53}$ erg calculated from 0 to $6 \mathrm{~s}$, $\eta=0.2, t_{\mathrm{p}}=6 \mathrm{~s}$, through Eq. (1) we estimate $\Gamma_{0} \sim 700 \pm 26$ $(520 \pm 20)$ in the case of a homogeneous medium with density $n=1 \mathrm{~cm}^{-3}\left(n=10 \mathrm{~cm}^{-3}\right)$. For a wind medium with $\dot{M}_{\mathrm{w}}=10^{-5} M_{\odot} \mathrm{yr}^{-1}$ and $v_{\mathrm{w}}=10^{3} \mathrm{~km} \mathrm{~s}^{-1}\left(v_{\mathrm{w}}=10^{2} \mathrm{~km} \mathrm{~s}^{-1}\right)$, following the relation $n_{0}=\dot{M}_{\mathrm{w}} / 4 \pi v_{\mathrm{w}} m_{\mathrm{p}}$, the initial bulk Lorentz factor is $\Gamma_{0} \sim 230 \pm 6(130 \pm 3)$. The errors are only statistical and were calculated using the uncertainties on the observables $E_{\text {iso }}$ and $t_{\mathrm{p}}$; the errors do not include the unknown uncertainties on parameters $\eta$ and $n_{0}$.

Table 2 in Ghirlanda et al. (2018) lists the coefficients that are required to calculate $\Gamma_{0}$ for all the other proposed formulae for the homogeneous and for the wind case. The resulting $\Gamma_{0}$ values differ at most by a factor of 2 . The computed values are similar to those found for other GBRs detected by LAT, which show a peak in the light curve in the LAT energy band (Ghirlanda et al. 2018).

\section{Discussion}

Our results indicate that a power-law component appears at $\sim 4$ $\mathrm{s}$ after trigger in the GBM data, that it peaks at $6 \mathrm{~s}$, and then declines. This temporal behavior matches that of the flux above $100 \mathrm{MeV}$, as seen by the LAT. Figure 2 shows that the emission in the two detectors (GBM and LAT) joins smoothly, with a consistent slope (within the errors). It is therefore compelling to interpret the two power laws seen in LAT and GBM as belonging to a single emission component. We propose that this nonthermal emission is produced by the external shock that is driven by the jet into the circum-burst medium. Its peak marks the jet deceleration time, that is, onset time of the afterglow.

The reasons leading to this interpretation are (i) they appear after the trigger of the prompt event, and peak when most of the prompt emission energy has already been radiated; (ii) they last much longer than the prompt emission; iii) they are characterized by a spectral index $\left(\Gamma_{\mathrm{PL}} \sim-2\right)$ typical of the known afterglows; (iv) with the exception of the early variable phases, their light curve smoothly decays with a temporal slope typical of the known afterglows.

We remark that this is not the first time that a power law is detected in the hard X-rays in addition to the spectral components that are usually seen during the prompt emission phase. A component like this was well visible in GRB 090202B, another burst that was very strong in the LAT band (Rao et al. 2013 and references above). The observation of the onset of the afterglow in the hard X-ray band is new, however, as is that it was found to be simultaneous within the uncertainties with the peak of the LAT light curve. This is especially important in this burst because of the MAGIC detection.

Our results imply that emission in the energy range between $10 \mathrm{keV}$ and $30 \mathrm{GeV}$ is produced by a single mechanism. If this mechanism is synchrotron or inverse Compton emission, this in turn implies that the energy of the underlying electron distribution must extend over more than three orders of magnitude.

We also know that the MAGIC telescope revealed photons above $300 \mathrm{GeV}$ (Mirzoyan et al. 2019) despite the strong absorption due to the extragalactic optical-infrared background (e.g., Franceschini et al. 2008) that is expected for $z=0.425$. If the maximum synchrotron energy is $h v_{\max }=m_{\mathrm{e}} c^{2} / \alpha_{\mathrm{F}} \sim 70 \mathrm{MeV}$ in the comoving frame, as theoretically predicted in the case of shock acceleration (Guilbert et al. 1983; de Jager et al. 1996), then the radiation above $300 \mathrm{GeV}$ might be interpreted as due to another process, most likely inverse Compton or synchrotron self-Compton emission. On the other hand, the observed maximum photon energy detected by LAT, $22.9 \mathrm{GeV} 15 \mathrm{~s}$ after trigger, does not violate the comoving $70 \mathrm{MeV}$ limit if the bulk Lorentz factor $\Gamma$ at this time is higher than 450 . For this value to be consistent with $\Gamma_{0}$, that is, the bulk Lorentz of the jet before it starts to be decelerated by the circum-burst medium, (assuming a prompt efficiency $\eta=0.2$ ) the circum-burst medium must not be too dense, with a number density $n \lesssim 30 \mathrm{~cm}^{-3}$ in the homogeneous case, or the progenitor stellar wind to be slightly faster and/or less massive than usually assumed, to satisfy $\dot{M}_{\mathrm{w},-5} v_{\mathrm{w}, 8} \lesssim 0.02\left(\right.$ where $\dot{M}_{\mathrm{w},-5}=\dot{M}_{\mathrm{w}} /\left(10^{-5} \mathrm{M}_{\odot} \mathrm{yr}^{-1}\right)$ and $\left.v_{\mathrm{w}, 8}=v_{\mathrm{w}} /\left(10^{8} \mathrm{~cm} \mathrm{~s}^{-1}\right)\right)$.

Alternatively, the entire spectral energy distribution from the $\mathrm{keV}$ to the $\mathrm{TeV}$ energy range could be inverse Compton emission, possibly by Compton scattering off IR-optical radiation. In this case, the MAGIC emission should connect smoothly with the LAT spectrum (i.e., it should not be harder). Therefore the MAGIC flux and spectrum will give crucial information about the origin of the entire high-energy spectrum of GRBs. 
Acknowledgements. We would like to thank Lara Nava for fruitful discussions. M. E. R. is grateful to the Observatory of Brera for the kind hospitality. This research has made use of data obtained through the High Energy Astrophysics Science Archive Research Center Online Service, provided by the NASA/Goddard Space Flight Center, and specifically, this work made use of public Fermi-GBM data. We acknowledge INAF-Prin 2017 (1.05.01.88.06) for support and the Italian Ministry for University and Research grant "FIGARO" 1.05.06.13. We also would like to thank for the support of the implementing agreement ASI-INAF n.2017-14-H.0.

\section{References}

Abdo, A. A., Ackermann, M., Asano, K., et al. 2009, ApJ, 707, 580 Ackermann, M., Asano, K., Atwood, W. B., et al. 2010, ApJ, 716, 1178 Ackermann, M., Ajello, M., Asano, K., et al. 2013, ApJS, 209, 11 Ackermann, M., Ajello, M., Asano, K., et al. 2014, Science, 343, 42 Aliu, E., Aune, T., Barnacka, A., et al. 2014, ApJ, 795, L3

Amati, L., Frontera, F., Tavani, M., et al. 2002, A\&A, 390, 81

Band, D., Matteson, J., Ford, L., et al. 1993, ApJ, 413, 281

Beardmore, A. P. 2019, GRB Coordinates Network, Circular Service, No. 23736, 23736

Beloborodov, A. M. 2005, ApJ, 627, 346

Beniamini, P., Nava, L., Duran, R. B., \& Piran, T. 2015, MNRAS, 454, 1073

Bošnjak, Ž., Daigne, F., \& Dubus, G. 2009, A\&A, 498, 677

Carosi, A., Antonelli, A., Becerra Gonzalez, J., et al. 2015, in 34th Internationa Cosmic Ray Conference (ICRC2015), eds. A. S. Borisov, V. G. Denisova, Z. M. Guseva, et al., Int. Cosmic Ray Conf., 34, 809

Castro-Tirado, A. J., Hu, Y., Fernandez-Garcia, E., et al. 2019, GRB Coordinates Network, Circular Service, No. 23708, 23708

Cerutti, B., Werner, G. R., Uzdensky, D. A., \& Begelman, M. C. 2013, ApJ, 770 147

Chevalier, R. A., \& Li, Z.-Y. 2000, ApJ, 536, 195

Daigne, F. 2012, Int. J. Mod. Phys. Conf. Ser., 8, 196

de Jager, O. C., Harding, A. K., Michelson, P. F., et al. 1996, ApJ, 457, 253

Del Monte, E., Barbiellini, G., Donnarumma, I., et al. 2011, A\&A, 535, A120

Franceschini, A., Rodighiero, G., \& Vaccari, M. 2008, A\&A, 487, 837

Frederiks, D., Golenetskii, S., Aptekar, R., et al. 2019, GRB Coordinates Network, Circular Service, No. 23737, 23737

Ghirlanda, G., Ghisellini, G., \& Nava, L. 2010, A\&A, 510, L7

Ghirlanda, G., Nava, L., Ghisellini, G., et al. 2012, MNRAS, 420, 483

Ghirlanda, G., Nappo, F., Ghisellini, G., et al. 2018, A\&A, 609, A112

Ghisellini, G., Ghirlanda, G., Nava, L., \& Celotti, A. 2010, MNRAS, 403, 926

Giuliani, A., Mereghetti, S., Fornari, F., et al. 2008, A\&A, 491, L25

Giuliani, A., Fuschino, F., Vianello, G., et al. 2010, ApJ, 708, L84

Goldstein, A., Burgess, J. M., Preece, R. D., et al. 2012, ApJS, 199, 19

González, M. M., Dingus, B. L., Kaneko, Y., et al. 2003, Nature, 424, 749

Gropp, J., Kennea, J. A., Klingler, N. J., et al. 2019, GRB Coordinates Network, Circular Service, No. 23688, 23688

Gruber, D., Goldstein, A., Weller von Ahlefeld, V. 2014, ApJS, 211, 12

Guilbert, P. W., Fabian, A. C., \& Rees, M. J. 1983, MNRAS, 205, 593
Hamburg, R., Veres, P., Meegan, C., et al. 2019, GRB Coordinates Network, Circular Service, No. 23707, 23707

He, H.-N., Wu, X.-F., Toma, K., Wang, X.-Y., \& Mészáros, P. 2011, ApJ, 733, 22

Hoischen, C., Balzer, A., Bissaldi, E., et al. 2017, Int. Cosmic Ray Conf., 35, 636 Hurley, K., Dingus, B. L., Mukherjee, R., et al. 1994, Nature, 372, 652

Kalberla, P. M. W., Burton, W. B., Hartmann, D., et al. 2005, A\&A, 440, 775

Kaneko, Y., Preece, R. D., Briggs, M. S., et al. 2006, ApJS, 166, 298

Kocevski, D., Omodei, N., Axelsson, M., et al. 2019, GRB Coordinates Network, Circular Service, No. 23709, 23709

Kumar, P., \& Barniol Duran, R. 2009, MNRAS, 400, L75

Kumar, P., \& Barniol Duran, R. 2010, MNRAS, 409, 226

Kumar, P., Hernández, R. A., Bošnjak, Ž., \& Barniol Duran, R. 2012, MNRAS, 427, L40

Lyutikov, M. 2010, MNRAS, 405, 1809

Meegan, C., Lichti, G., Bhat, P. N., et al. 2009, ApJ, 702, 791

Minaev, P., \& Pozanenko, A. 2019, GRB Coordinates Network, Circular Service, No. 23714, 23714

Mirzoyan, R., Noda, K., Moretti, E., et al. 2019, GRB Coordinates Network, Circular Service, No. 23701, 23701

Molinari, E., Vergani, S. D., Malesani, D., et al. 2007, A\&A, 469, L13

Nava, L. 2018, Int. J. Mod. Phys. D, 27, 1842003

Nava, L., Ghirlanda, G., Ghisellini, G., \& Celotti, A. 2011, A\&A, 530, A21

Nava, L., Sironi, L., Ghisellini, G., Celotti, A., \& Ghirlanda, G. 2013, MNRAS, 433, 2107

Nava, L., Vianello, G., Omodei, N., et al. 2014, MNRAS, 443, 3578

Nava, L., Desiante, R., Longo, F., et al. 2017, MNRAS, 465, 811

Oganesyan, G., Nava, L., Ghirlanda, G., \& Celotti, A. 2017, ApJ, 846, 137

Oganesyan, G., Nava, L., Ghirlanda, G., \& Celotti, A. 2018, A\&A, 616, A138

Omodei, N. 2009, in AIP Conf. Ser., eds. D. Bastieri, \& R. Rando, 1112, 8 Panaitescu, A. 2017, ApJ, 837, 13

Rao, A., Basak, R., Bhattacharya, J., et al. 2013, Res. Astron. Astrophys., 14

Ravasio, M. E., Oganesyan, G., Ghirlanda, G., et al. 2018, A\&A, 613, A16

Romano, P., Campana, S., Chincarini, G., et al. 2006, A\&A, 456, 917

Sari, R., \& Piran, T. 1999, ApJ, 520, 641

Selsing, J., Fynbo, J. P. U., Heintz, K. E., et al. 2019, GRB Coordinates Network, Circular Service, No. 23695, 23695

Sommer, M., Bertsch, D. L., Dingus, B. L., et al. 1994, ApJ, 422, L63

Toma, K., Wu, X.-F., \& Mészáros, P. 2011, MNRAS, 415, 1663

Ursi, A., Tavani, M., Marisaldi, M., et al. 2019, GRB Coordinates Network, Circular Service, No. 23712, 23712

Uzdensky, D. A., Cerutti, B., \& Begelman, M. C. 2011, ApJ, 737, L40

Wang, Y., Li, L., Moradi, R., \& Ruffini, R. 2019, ArXiv e-prints [arXiv:1901.07505]

Wilms, J., Allen, A., \& McCray, R. 2000, ApJ, 542, 914

Xiao, S., Li, C. K., Li, X. B., et al. 2019, GRB Coordinates Network, Circular Service, No. 23716, 23716

Yassine, M., Piron, F., Mochkovitch, R., \& Daigne, F. 2017, A\&A, 606, A93

Yonetoku, D., Murakami, T., Nakamura, T., et al. 2004, ApJ, 609, 935

Zhang, B.-B., Zhang, B., Liang, E.-W., et al. 2011, ApJ, 730, 141 